\title{
Satellite Breakup Risk Mitigation
}

\author{
Darrin P. Leleux ${ }^{1}$ and Jason T. Smith ${ }^{2}$ \\ Orbit Dynamics Branch, Johnson Space Center, Houston TX 77058
}

\begin{abstract}
Many satellite breakups occur as a result of an explosion of stored energy on-board spacecraft or rocketbodies. These breakups generate a cloud of tens or possibly hundreds of thousands of debris fragments which may pose a transient elevated threat to spaceflight crews and vehicles. Satellite breakups pose a unique threat because the majority of the debris fragments are too small to be tracked from the ground. The United States Human Spaceflight Program is currently implementing a risk mitigation strategy that includes modeling breakup events, establishing action thresholds, and prescribing corresponding mitigation actions in response to satellite breakups.
\end{abstract}

\section{Nomenclature}

EVA $\quad=$ Extra-Vehicular Activity

ISS $\quad=$ International Space Station

LEO $\quad$ Low Earth Orbit

MMOD $\quad=$ Micrometeoroid/Orbital Debris

NASA $\quad=$ National Aeronautics and Space Adminstration

ORA $\quad=$ Optimal Relative Attitude; An inertial protection attitude which aligns the least debrissensitive element of the asset with the relative velocity vector between the asset and the debris cloud.

RADAR $\quad=$ Radio Detection and Ranging

SBRAM $\quad=$ Satellite Breakup Risk Assessment Model

SSN $\quad=$ Space Surveillance Network

TCA $=$ Time of Closest Approach

USSTRATCOM = United States Strategic Command

\section{Introduction}

Satellite breakups happen for a variety of reasons. Many breakups involve an explosion of stored energy aboard active and inactive rocketbodies and spacecraft. Other breakups are the result of atmospheric-induced heating on debris pieces close to reentering the Earth's atmosphere or collisions between debris pieces. As a result of these events, a cloud containing tens or possibly hundreds of thousands of debris fragments is generated. Due to atmospheric drag and perturbations caused by the Earth's oblateness, this cloud will spread throughout the orbital plane of the parent object and eventually dissipate into the background space environment over a period of several weeks. Previous studies have shown that $70 \%$ of breakups occur in the area of space occupied by human spaceflight activities ${ }^{1}$.

The debris cloud generated by a satellite breakup poses a transient elevated risk of collision above the background orbital debris environment for any vehicle whose orbit intersects that of the debris cloud. Historically, satellite breakups average 4-5 events per year and most are not a threat to human space operations. However,

${ }^{1}$ Corresponding author (darrin.p.leleux@nasa.gov); Flight Dynamics Officer (FDO), Mail Code: DM32, NASA Johnson Space Center, Houston TX, 77058, AIAA Member. A United Space Alliance, LLC employee performing a Government Accountable Function.

${ }^{2}$ Trajectory Operations Officer (TOPO), Mail Code: DM33, NASA Johnson Space Center, Houston TX, 77058.

American Institute of Aeronautics and Astronautics 
approximately $10 \%$ of the breakups over the past 6 years have created enough risk in terms of probability of collision with human spaceflight vehicles to cause concern ${ }^{2}$. This study attempts to quantify the risk posed by satellite breakups to NASA's Human Spaceflight vehicles and crews and provide action thresholds and corresponding rationale for the action thresholds. The study will also define mitigating actions and characterize their effectiveness.

\section{Calculating the Risks}

For the most part, the debris fragments generated by a high-energy satellite breakup are too small to be tracked using current ground-based tracking capabilities; however, some trackable pieces of debris are generated. It is by tracking these larger debris pieces that a breakup is recognized. Notification that a satellite breakup has occurred is provided by USSTRATCOM through the use of the tracking resources of the SSN. A breakup is identified when ground tracking RADAR observes a single cataloged space object as having multiple pieces where one piece was expected.

High-energy satellite breakups provide a unique threat to human space operations because of the untrackability of the majority of the debris fragments. However, although most of the individual pieces of debris are not trackable, the composition and propagation of the debris cloud can be predicted using the SBRAM software ${ }^{3}$. This tool was developed by NASA's Orbital Debris Program Office at the Johnson Space Center. It performs a Monte Carlo simulation to characterize a satellite breakup and develop a probability of catastrophic collision between the debris cloud and the vehicle of interest at each possible orbital intersection. Summing these individual probabilities gives a cumulative probability of catastrophic collision due to the breakup over a defined period of time.

Aside from the geometry of the orbit intersections, SBRAM considers the exposed areas of the vehicle and the susceptible particle diameters for each corresponding vulnerable area. By filtering out the particles from which the vehicle is shielded and considering the size of the original vehicle, SBRAM can calculate the probabilities of catastrophic collision for various vehicles, vehicle configurations or EVA crewmembers. A subtle point here is that the particle diameters which are chosen for consideration are those that have the potential to cause a catastrophic collision with the asset at orbital velocities. Thus smaller particles which have the potential to cause damage, but not catastrophic damage, are not considered in the probability calculations. These particle sizes are all vehicle specific and should be determined through experimentation by firing various size particles at hypervelocity at mockups of flight hardware to determine these thresholds. For the Shuttle and ISS, these studies were performed by the Hypervelocity Impact Technology Facility at the Johnson Space Center. The details of the SBRAM software are described in Ref. 3; although, some updates have been made to the software since this paper was published.

Some limitations of calculating the risks due to satellite breakups are the availability and timeliness of breakup data. As mentioned earlier, the recognition that a satellite breakup has occurred relies on the availability of a tracking RADAR. Depending on where the object broke up with respect to the Earth's surface and the availability of SSN resources in that area, a breakup may not be recognized for some time after the event. In addition, it is possible that by the time the breakup cloud passes within range of a RADAR the trackable pieces might have separated by a sufficient distance so as not to be recognized as associated with the same object and thus as a breakup. This can present a great deal of uncertainty to the decision maker. A method to deal with this uncertainty is based on establishing validity criteria for the probabilities calculated by SBRAM. These criteria will dictate when enough information is available to be able to reliably model the satellite breakup. If the information provided does not meet the validity criteria, for example, a large uncertainty of the time of the breakup, then no action is required for this event, although some precautions may be prudent.

\section{Action Thresholds}

Two of the key elements of any risk mitigation strategy are determining when action should be taken and what elements of risk must be controlled. A typical approach is to weigh the likelihood of the event occurring versus the consequence. With satellite breakups there are two elements of risk that need to be controlled including the individual encounter with the debris cloud and the cumulative risk. For both of these risk elements, the 
consequence of a collision occurring would be catastrophic, since that is how the probabilities are defined given the particle sizes mentioned in the previous section. Thus the primary variable to determine when action should be taken is the likelihood, and the likelihood is represented by the probability of collision. Thus, for each of the elements listed above, an action threshold must be defined.

The first element is the individual encounter with the debris cloud. The risk due to each individual encounter should be kept below some threshold. The second element is the cumulative risk due to repeated encounters with the debris cloud. While no individual encounter may exceed a given threshold, the cumulative risk may be high, thus exposing the vehicle and crew to an extended period of increased risk.

In choosing action thresholds, three general methods for proposing rationale from which to base a threshold have been identified. Each type of rationale is appropriate in particular circumstances. They are as follows:

1. Choose a threshold which is based on precedence. This method is based on currently accepted risk levels and assumes these levels have valid rationale.

2. Choose a threshold which is based on the percentage increase over and above that which has already been accepted.

3. Choose a threshold which balances the overall risk reduction with the number of times a mitigation action may need to be taken should that threshold be chosen. This typically involves studying historical data or a current database of objects and predicting how many threshold violations may occur given a proposed threshold.

\section{A. Individual Encounters}

As mentioned earlier, the first element of risk to be controlled is the individual encounter with the debris cloud. This element of risk involves the asset traversing through a particularly dense orbital intersection at a specific instant in time increasing the risk to the asset. This type of risk is very similar to the type of risk involved with an individual encounter with a trackable piece of debris in that a distinct time is identified which poses a threat to the asset. This provides justification to choose an action threshold which is the same as that which was chosen for the trackable debris risk threshold since the consequence of a collision from either of these events is the same. In the case of the Shuttle and ISS programs, this risk level was chosen to be $10^{-4}$ or 1 in 10,000 chance of catastrophic collision. The primary risk mitigation action for trackable debris is a translational maneuver to avoid the object; therefore, the original threshold was chosen to balance the risk reduction by performing the maneuver with the number of times that a translational maneuver would have to be performed given a certain population of trackable debris. Thus, the risk threshold for trackable debris has precedence and valid rationale. When determining the threshold in the case of satellite breakups, the same 1 in 10,000 chance of catastrophic collision was chosen as an action threshold for the individual encounter between the asset and the debris cloud.

\section{B. Repeated Encounters}

The second element of risk that needs to be controlled is the repeated encounter with the debris cloud. This element of risk is different than the individual encounter in that there are no individual orbital intersections which have been identified as particularly risky, but there are a large number of encounters with the debris cloud which cumulatively add up to an unacceptable risk level. An example of this might be a breakup which occurred in an orbit very similar to the asset where an orbital intersection occurs every half orbit. In choosing an action threshold for this risk element, it was found that this type of risk was very similar to the type of risk involved with the background flux of orbital debris (MMOD) albeit at higher density levels. With MMOD, human spaceflight risk thresholds are based on an ISS program-level decision to keep cumulative risk below 5\% or 1 in 20 over 10 years. This risk level has become the basis for determining satellite breakup risk thresholds for repeated encounters; however, a different approach was required between Shuttle and ISS.

The 1 in 20 over 10 years limit translates into a $1.4 \times 10^{-4}$ risk level per 10-day period. A 10-day period was chosen to accumulate risk instead of a daily limit since this provides a longer period of time over which to average the probabilities. After reviewing historical records back to the launch of the first ISS element, it was found that if a $1 \times 10^{-4}$ level of risk per 10-day period was chosen, two satellite breakups would have violated this threshold ${ }^{2}$. Figure 1 shows a graph of this tradeoff by choosing different thresholds and seeing how many times these thresholds would have been exceeded during the 6.5 years considered in the historical study. A threshold of $1 \times 10^{-}$

American Institute of Aeronautics and Astronautics 
${ }^{4}$ per 10-day period was chosen as a compromise between risk increase and expected action frequency.

Since the duration of Shuttle missions is very short compared to the ISS (traditionally less than 12 days), the rationale for choosing a cumulative threshold for repeated encounters must be different. Because the mission is of defined length, the total cumulative risk due to a breakup can be compared to the MMOD risk already accepted as a result of flying a mission. For the Shuttle Program, keeping the cumulative risk due to MMOD to less than 1 in 1,000 chance of debris strike is the goal. This value was derived and adopted from the Station risk limit after the Columbia accident. This is seldom achieved due to changing criteria for damage tolerance on critical elements of the Shuttle as well as unfavorable vehicle attitudes which must be flown to accomplish the mission. Be that as it may, a 1 in 1,000 risk is the goal that is used as the basis for choosing an action threshold for satellite breakups. The risk posed by a satellite breakup is over and above that which was accepted prior to mission launch. The finite duration of Shuttle missions lends itself to the use of the second method of choosing an action threshold mentioned before, i.e. choose a threshold which is based on the percentage increase over and above that which has already been accepted $\left(1\right.$ in 1,000). This means that if an action threshold is chosen to be 1 in $10,000\left(1 \times 10^{-4}\right)$ then this indicates a $10 \%$ increase in the total MMOD risk from that which was accepted pre-flight. After discussion with mission managers, the $1 \times 10^{-4}$ value was deemed appropriate and acceptable.

\section{Historical Study (Nov. 98 - Jun. 05)}

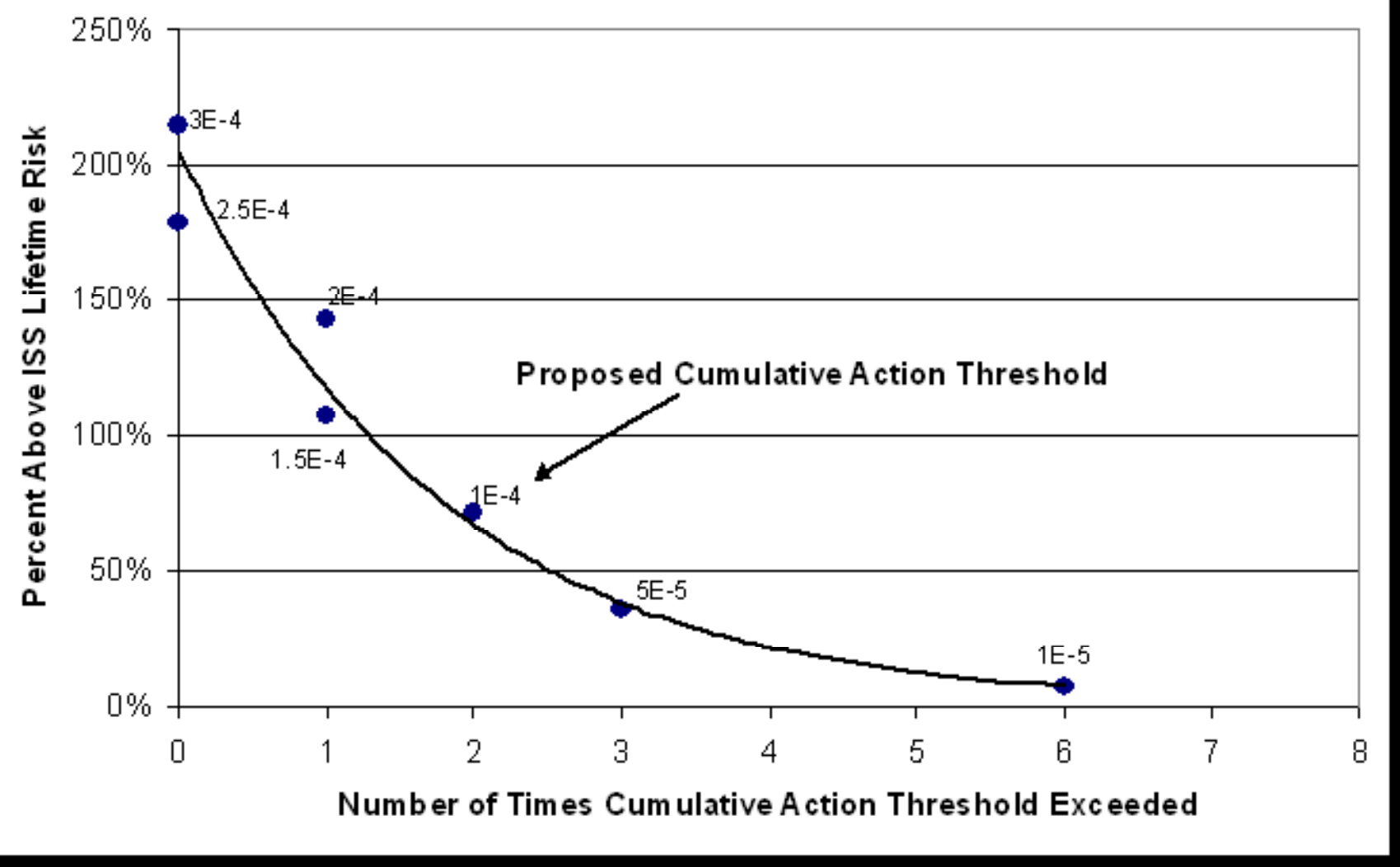

Figure 1. Historical Study (November 1998 - June 2005). This figure details a historical study indicating how many times a proposed threshold would have been violated if a particular action threshold would have been chosen. It also indicates what the percent increase risk would have been above the ISS MMOD program-level lifetime 10-day risk threshold. The solid line is a fit showing the general trend of the data. 


\section{Mitigation Actions}

Once action thresholds have been defined, the next step is to identify appropriate actions which can be taken to mitigate the risk. Each action should also be quantified with how much risk reduction is provided by performing the action. Some actions are more appropriate than others in certain circumstances. In the case of breakups, the actions should be categorized in terms of the two risk elements, individual encounters and repeated encounters.

The actions which have been identified as appropriate for satellite breakups are a combination of actions which are used for traditional avoidance of trackable debris ${ }^{4}$ and un-trackable MMOD. The following actions are presented as examples of possible risk mitigation techniques. Advantages and disadvantages for each example are also discussed.

\section{A. Optimal Relative Attitude}

As explained above, the probability of catastrophic collision is a function not only of the orbital geometry, but also the exposed area of the vehicle and the minimum size particle which will cause catastrophic damage to the vehicle. The ORA, aligns the least debris-sensitive areas of the vehicle with the relative velocity vector between the vehicle and the debris cloud. For LEO encounters, this attitude is generally a yaw relative to the vehicle body axes.

Figure 2 shows an example satellite breakup and the evolution of the approach geometry between the ISS and the debris from the breakup of object 20390 which occurred on April 18, 1999. The top graph indicates the pitch angle as seen from the ISS of the approaching debris piece. These angles are all very small which will be the case for LEO close approaches as mentioned previously. This indicates that the encounters will take place essentially in the Local Horizontal plane of the ISS. The middle graph indicates the Yaw angle from which the debris will appear to approach. This angle may change over time due to differential nodal regression experienced by the ISS and the debris cloud as a result of differing orbits. This is indicated by the slope of the line indicating the yaw angles. The other item that can be seen from the middle graph is the fact that approaches will be from both the right as well as the left with respect to the ISS indicated by both positive and negative yaw angles. Finally in the bottom graph, the reader can see the distribution of right and left approaches with respect to how risky each might be. During the first four days of this example breakup, only right approaches exceeded a risk of $10^{-6}$.

The ORA has the potential to reduce the probability in two ways. First, by exposing the best shielded sections of the vehicle to the debris cloud, the catastrophic probability is decreased. Second, by minimizing the cross sectional area exposed to the debris cloud, the probability of collision is reduced. For the Shuttle and ISS, it happens to be the case that the vehicle attitude that presents the least debris-sensitive elements to the debris also has the least cross-sectional area. Use of the ORA is advantageous in several ways. It is a relatively low impact to mission operations to change the vehicle attitude as opposed to performing a translational maneuver. Also, the ORA reduces the probability in the near term, instead of delaying the encounter until a future time which would be the case for a translational maneuver.

The ORA also has some potential disadvantages. First, depending on vehicle capabilities, the required attitude may be outside of the approved attitude envelope. Second, if there are multiple high-risk encounters with the debris cloud, several attitude maneuvers may be required, or it may be required to hold a non-optimal attitude for a long period of time. Either of these reasons could potentially prohibit the use of the ORA, or make its use more risky than the benefit gained.

American Institute of Aeronautics and Astronautics 
Satellite Breakup Approach Geometry (Sat No. 20390)
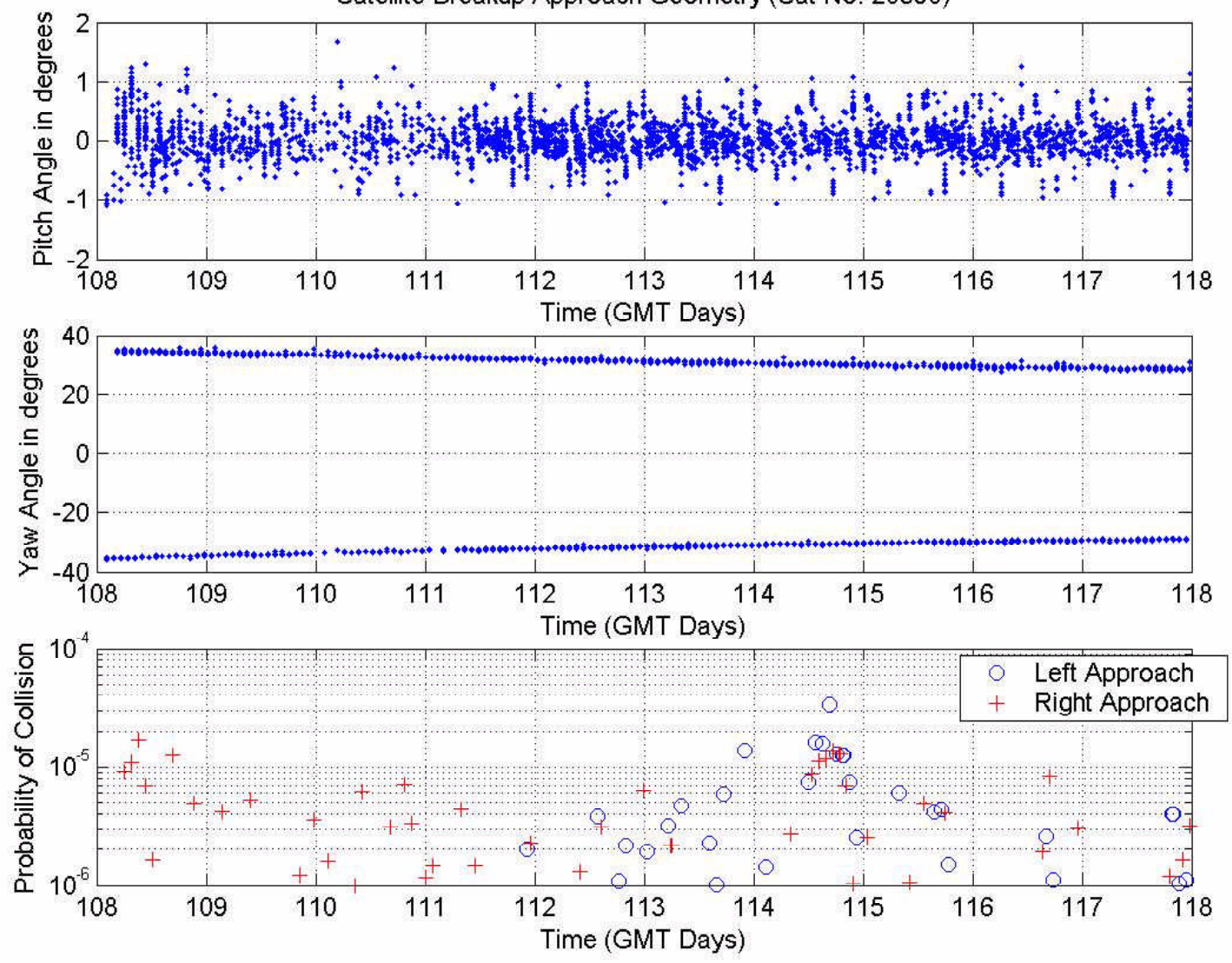

Figure 2. Evolution of Approach Geometry. This figure shows the evolution of the approach geometry between an example breakup of object 20390 and the ISS. Each data point represents a close approach between the ISS and a debris piece from object 20390 as modeled by SBRAM.

\section{B. Translational Maneuver}

A second risk mitigation action would be to use a translational maneuver. This maneuver would be designed so as to reduce the probability of collision of a particular high-risk encounter with the debris cloud. The advantage of this action is a reduction of the probability at a specific period of time using an established procedure. However, there are several disadvantages of this action as well. Previous studies have shown that translational maneuvers have a tendency to reduce one high-risk encounter at the expense of increasing the risk of a subsequent encounter. ${ }^{2}$ Thus, the threat is not eliminated, but merely delayed. For vehicles which will remain on-orbit for a short period of time, it may be possible to delay the time of the high-risk encounter until after the vehicle has completed its mission. However, for vehicles remaining on-orbit for extended periods, this action is unlikely to eliminate the threat, but rather delay the encounter. Further, translational maneuvers have been shown to have little effect on the cumulative probability, and thus should not be used to reduce this type of risk.

\section{Mission Timeline}

A third possible mitigation action involves changing the mission timeline. Specifically, this would entail delaying a launch or expediting a landing. The goal with this action is to avoid high-risk periods entirely, thus eliminating the risk or greatly reducing it. There is the potential that this action, particularly delaying a launch, could have very minor impacts but yield sustantial reduction of risk. However, in the case of an early landing, 
there could be substantial mission objectives which could be left unaccomplished. In the event that this would cause an additional future mission, this risk is likely to outweigh the reduction in risk gained by an early landing. Finally, for long-duration missions, an early landing may not be possible.

\section{Vehicle Inspection}

A forth mitigating action involves performing an inspection of the vehicle following a high-risk encounter with the debris cloud. Due to the small particle size of the debris particles associated with a satellite breakup, in certain instances it is possible to suffer catastrophic damage without an immediate awareness of such damage. A primary example of this would be damage to the thermal protection system. This damage is difficult to detect on-orbit but can prove catastrophic during entry. By performing an inspection, the risk associated with past encounters with the debris cloud can be eliminated, thus resetting the cumulative probability back to zero.

This action provides unique advantages in that it allows for a response to past breakups for which timely notification was not available. In addition, it provides a response in instances when there is insufficient time prior to encounters with the debris cloud to perform other actions listed. However, this action is not preferred due to its reactive, instead of proactive nature. Also, this action depends on having the necessary time and vehicle resources to perform these inspections.

\section{E. Crew Activity}

This action specifically applies to crewed vehicles. In cases where certain parts of the vehicle provide better shielding than others, the crew can take shelter in the areas of better shielding during high-risk encounters with the debris cloud. Due to the small size of most of the debris particles generated during a satellite breakup, it is most likely, even in cases of high risk of catastrophic collision, that a debris strike on the vehicle would not create a rapid depressurization. Instead, vehicle shielding offers protection in these instances. Placing the crew in an area of greatest shielding minimizes their risk during encounters with the debris cloud.

The obvious advantage of this action is crew safety, which should be the primary concern of every crewed vehicle. Also, this action requires little lead time and is thus available in situations where notification of a breakup is delayed or other scenarios where there is insufficient time to perform other actions. However, as with the inspection activity above, this action is reactive and does nothing to avoid the debris cloud or reduce the probability of collision. Also, depending on the timing of the encounter, there may be significant impediments to the crew taking shelter including such things as crew sleep, robotics operations, and EVA.

\section{F. Array Positioning}

This action pertains to the positioning of solar arrays or radiators in the event of a high-risk encounter with the debris cloud. This action is highly dependent on the vehicle configuration and capabilities. There are two basic strategies for solar arrays and other vehicle appendages during a high risk encounter with the debris cloud. The first is to feather the arrays, or minimize the area exposed to the debris cloud. This action will offer the greatest protection for the arrays. A second, contradictory option is to position the arrays so as to best shield other parts of the vehicle from the debris cloud. This action potentially sacrifices the arrays in order to protect more sensitive parts of the vehicle.

A decision as to whether to feather the arrays or use them for shielding must be made on a vehicle by vehicle basis. The goal is to minimize the overall risk to the vehicle and crew. In some cases this can be accomplished by increasing the risk to the arrays in order to decrease the risk to more sensitive areas, including pressurized volumes. In other cases, the risk of loss of array performance may outweigh the benefits of increased shielding. In either case, positioning of the arrays may create adverse impacts to power generation or heat rejection which could outweigh either of the previous benefits. A decision as to which action is most appropriate must be made for each specific vehicle and should consider the risk of the satellite breakup as well as the risk of performing a particular action. 


\section{G. Extra-Vehicular Activity}

This action refers to EVA during a satellite breakup. The calculation of the probability of catastrophic collision for EVA should use appropriate areas and particle sizes which are most likely different than those used for the vehicle. If the EVA probability violates a threshold during the time of an EVA, the only action to take is to reschedule the EVA. If the EVA is already in progress, it should be terminated. These actions should be performed in addition to any of the above actions pertaining to the vehicle if there is also a violation of the vehicle probability threshold. Finally, if there is a violation of the vehicle probability but not of the EVA, consideration should be given to rescheduling the EVA anyway. The risk of canceling an EVA is primarily a schedule risk as opposed to vehicle or crew risk posed by a satellite breakup event.

\section{Conclusion}

Satellite Breakups can present a transient elevated threat to Human Spaceflight vehicles and crews. The proposed new method establishes a risk mitigation strategy for Human Spaceflight operations and makes use of all available information to recommend consistent and appropriate actions due to calculated risks. Although this strategy has been developed for human spaceflight, with some vehicle-specific modifications, these concepts can be extended to unmanned operations. The basic process of identifying the action thresholds and possible mitigation actions is still valid. Currently, the biggest limitation to the process is the availability and timeliness of breakup information as discussed in section II. Despite this limitation, the process identified in this paper provides a logical framework from which decisions can be made with respect to satellite breakups.

\section{Acknowledgments}

The authors would like to acknowledge the support of their employers the United Space Alliance and the National Aeronautics and Space Administration. They would also like to express gratitude to the NASA Orbital Debris Program Office for the opportunity to exchange ideas and for developing the SBRAM software.

\section{References}

${ }^{1}$ Theall, J. R., and Matney, M. J., "Near-Term Effects From Satellite Breakups on Manned Space Activities," AIAA 38th Aerospace Sciences Meeting \& Exhibit, AIAA-2000-0763, Reno, NV, 10-13 January 2000, pg.3.

${ }^{2}$ Holmes, L., "Analysis of Past and Possible Threats Posed by Satellite Breakups and Potential Actions to Minimize Their Risk to Human Tended Vehicles,” NASA/CR-2005-213691, October 2005.

${ }^{3}$ Matney, M. J., and Theall, J. R., "The Use of the Satellite Breakup Risk Assessment Model (SBRAM) to Characterize Collision Risk to Manned Spacecraft," IAA Paper 99-IAA.6.5.09, October 1999.

${ }^{4}$ Leleux, D. P., et. al., "Probability-Based Space Shuttle Collision Avoidance," SpaceOps 2002 Conference, Houston TX, October 2002.

American Institute of Aeronautics and Astronautics 